\title{
Prueba empírica de un modelo de calidad de vida ${ }^{1}$
}

\section{Empirical test of a model of quality of life \\ Recibido: 19 de febrero de 2014 - Revisado: 7 de noviembre de 2014 - Aceptado: 25 de marzo de 2015}

Erle García Estrada ${ }^{2}$ - Cruz García Lirios ${ }^{3}$ - José Francisco Rosas Ferrusca ${ }^{4}$ María Beatriz Castillo Escamilla ${ }^{5}$ - Javier Carreón Guillén 6

Jorge Hernández Valdés ${ }^{7}$ - Bertha Leticia Rivera Varela ${ }^{8}$

\section{Resumen}

El presente trabajo se propuso establecer un modelo correlativo para discutir la importancia de otras variables en la investigación de la calidad de vida. Una vez se especificaron las relaciones entre los factores derivados de la revisión de la literatura, se llevó a cabo un estudio transversal con una muestra no probabilística de 245 estudiantes. Cuando se obtuvo la validez y confiabilidad del instrumento que midió: satisfacción de vida, capacidades esperadas, expectativas de oportunidad, relaciones de confianza, percepción de justicia, valoración del entorno, normas de contexto y recursos percibidos, se contrastó un modelo estructural $\left[\mathrm{X}^{2}=12,35(12 \mathrm{gl}) ; \mathrm{p}=0,000 ; \mathrm{GFI}=0,975 ; \mathrm{RMR}=0,000\right]$ en el que la percepción de disponibilidad de recursos correlacionó indirectamente a la satisfacción de vida a través de las normas de contexto $(\gamma=0,52)$. Los resultados se compararon con los hallazgos reportados en el estado del conocimiento.

\section{Palabras clave}

Calidad de vida, satisfacción vital, normas grupales, disponibilidad de recursos, capacidades percibidas.

\section{Abstract}

This work was proposed to establish a correlative model to discuss the importance of other variables in the investigation of the quality of life. Once the relationship between the factors derived from the literature review were specified, a crosssectional study was carried out with a nonrandom sample of 245 students. When the validity and reliability of the instrument measured was obtained: life satisfaction, expected capacities, expectations of opportunity, trust relationships, perceptions of fairness, valuing the environment, rules of context and perceived resources, a structural model was contrasted $\left[\mathrm{X}^{2}=12,35(12 \mathrm{gl}) \mathrm{p}=0.000 ; \mathrm{GFI}=\right.$ $0.975 ; \mathrm{RMR}=0.000]$ in which the perceived availability of resources indirectly correlated to life satisfaction through context rules $(\gamma=0.52)$. The results were compared with the findings reported in the state of knowledge.

\section{Keywords}

Quality of life, life satisfaction, group norms, availability of resources, perceived capabilities.

\footnotetext{
Investigación del proyecto: Gobernanza de los estilos de vida sustentables y las identidades de género, auspiciada por la Red de Estudios Transdisciplinares UAEMEXUNAM, México.

${ }^{2}$ M.Sc. en Educación, profesora en Universidad Autónoma del Estado de México (UAEM)-Unidad Académica Profesional Huehuetoca (UAPH), Huehuetoca, México. Correo electrónico: egarciae@uaemex.mx

${ }^{3}$ Ph.D. (c) en Psicología Social y Ambiental, profesor en Universidad Autónoma del Estado de México (UAEM)-Unidad Académica Profesional Huehuetoca (UAPH), Huehuetoca, México. Correo electrónico garcialirios@yahoo.com

${ }^{4}$ Ph.D. en Administración Pública, profesor en Universidad Autónoma del Estado de México (UAEM)-Unidad Académica Profesional Huehuetoca (UAPH), Huehuetoca, México. Correo electrónico: jfrosasf@uaemex.mx

${ }^{5}$ M.Sc. en Educación, profesora en Escuela Nacional de Trabajo Social (ENTS) de la Universidad Nacional Autónoma de México (UNAM), México D. F., México. Correo electrónico: e_remo@terra.com

${ }^{6} \mathrm{Ph} . \mathrm{D}$. en Administración, profesor en Escuela Nacional de Trabajo Social (ENTS) de la Universidad Nacional Autónoma de México (UNAM), México D. F., México. Correo electrónico: javierg@unam.mx

${ }^{7}$ M.Sc. en Educación, profesor en Escuela Nacional de Trabajo Social (ENTS) de la Universidad Nacional Autónoma de México (UNAM), México D. F., México. Correo electrónico: jorheval@unam.mx

${ }^{8}$ Ph.D. en Geografía, profesora en Universidad Autónoma del Estado de México (UAEM)-Unidad Académica Profesional Huehuetoca (UAPH), Chimalhuacán, México. Correo electrónico: briveravarela@yahoo.com.mx

Para citar este artículo: García, C., Carreón, J., Hernandez, J., Rivera, B., Castillo, M., García, E., \& Rosas, J. (2015). Prueba empírica de un modelo de calidad de vida. Revista Civilizar Ciencias Sociales y Humanas, 15(28), 181-196.
} 


\section{Introducción}

La calidad de vida entendida como satisfacciones relacionadas con recursos, valores, normas, expectativas, percepciones y capacidades es un tema central en la agenda de salud pública para el desarrollo local sustentable (García, 2013).

Algunos estudios relativos a la calidad de vida (véase tabla 1) establecen diferencias significativas entre sexos con respecto a transporte, empleo y recreación (Baldi \& García, 2010). Así mismo, cuando se pondera como un sistema perceptual de los recursos alrededor del individuo y en referencia al grupo primario, se considera un estilo de bienestar personal que se orienta a la integración social (Barranco, Delgado, Meli, \& Quintana, 2010).

Empero, la calidad de vida se correlaciona con la ansiedad y la depresión en situaciones de incertidumbre médica y deterioro de la salud (Machado, Anarte, \& Ruiz, 2010). Es decir, la calidad de vida es el resultado de la percepción de escasez de recursos más que de las esperanzas generadas a partir de las capacidades personales. Las oportunidades parecen reducirse a su mínima expresión y con ello la responsabilidad de autogestión se incrementa y se observa un mejoramiento de expectativas a través de los nexos entre los integrantes de un grupo social, familiar o escolar.

Grimaldo (2010) encontró seis dimensiones alusivas a la calidad de vida. Se trata del bienestar económico, la relación interpersonal de pareja, situación familiar, contexto de vecindario, capital social y estado de salud. Por consiguiente, la calidad de vida es un entramado de expectativas que parten de un núcleo figurativo para incidir en las decisiones de distribución de recursos (Carreón \& García, 2013).

Cuando el individuo atraviesa por una coyuntura económica, sanitaria, familiar o interpersonal, a menudo estima que su calidad de vida se ha modificado sustancialmente (Sadeghzadeh, 2012). De inmediato se activan expectativas estéticas, afectivas y racionales que impulsan acciones que se dirigen a la generación de oportunidades y actualización de capacidades del sujeto para con el grupo al que pertenece o quiere pertenecer (Derya, 2012). Esto es, la calidad de vida es antecedente de la formación de una identidad de grupo y sentido de pertenencia anclada a libertades de elección, expectativas de justicia y movilización colectiva.

Tabla 1.

Estado del conocimiento de las variables correlacionadas con la calidad de vida

\begin{tabular}{|c|c|c|c|c|c|}
\hline Año & Autores & Constructo & Muestra & Instrumentos & Resultados \\
\hline 2010 & Baldi y García & $\begin{array}{l}\text { Calidad de vida: "hace referen- } \\
\text { cia a estilos o formas de vida, } \\
\text { o bien, a nivel estándar de vida } \\
\text { (consumo de bienes)" (Baldi y } \\
\text { García, 2010, p. 21) }\end{array}$ & $\begin{array}{l}168 \text { residentes } \\
\text { de la ciudad de } \\
\text { San Luis }\end{array}$ & $\begin{array}{l}\text { World Health Organiza- } \\
\text { tion Quality of Life }\end{array}$ & $\begin{array}{l}\text { Existen diferencias entre hombres } \\
\text { y mujeres con respecto a su grado } \\
\text { de satisfacción de calidad de vida; } \\
\text { transporte }\left(X^{2}=12,21, p=0,02\right) \text {, } \\
\text { energía y fatiga }\left(X^{2}=10,48, p=\right. \\
0,03), \text { capacidades laborales }\left(X^{2}=\right. \\
23,44, p=0,02) \text { y oportunidades } \\
\text { para recreación }\left(X^{2}=24,03, p=\right. \\
0,02)\end{array}$ \\
\hline
\end{tabular}




\begin{tabular}{|c|c|c|c|c|c|}
\hline Año & Autores & Constructo & Muestra & Instrumentos & Resultados \\
\hline 2010 & $\begin{array}{l}\text { Barranco, } \\
\text { Delgado, } \\
\text { Melin y } \\
\text { Quintana }\end{array}$ & $\begin{array}{l}\text { - Calidad de vida y bienestar } \\
\text { emocional aluden a sentirse } \\
\text { tranquilo, seguro, sin agobios y } \\
\text { no estar nervioso. } \\
\text {-Relaciones interpersonales } \\
\text { aluden a relacionarse con } \\
\text { distintas personas, tener amigos } \\
\text { y llevarse bien con la gente. } \\
\text { - Bienestar material es tener } \\
\text { suficiente dinero para comprar } \\
\text { lo que se necesita y se desea } \\
\text { tener, contar con una vivienda y } \\
\text { lugar de trabajo adecuados. } \\
\text { - Desarrollo personal es la po- } \\
\text { sibilidad de aprender diferentes } \\
\text { cosas, poseer conocimientos y } \\
\text { realizarse personalmente. } \\
\text { - Bienestar fisico se vincula con } \\
\text { gozar de buena salud, sentirse en } \\
\text { una buena forma fisica y tener há- } \\
\text { bitos de alimentación saludables. } \\
\text { - Autodeterminación es decidir } \\
\text { por sí mismo y tener oportuni- } \\
\text { dad de elegir cómo se quiere la } \\
\text { vida, el trabajo, el tiempo libre, } \\
\text { el lugar donde se habita y las } \\
\text { personas con las que se está. } \\
\text { - Integración social se conecta } \\
\text { con ir a lugares de la ciudad o } \\
\text { del barrio donde asisten otras } \\
\text { personas y participar de sus } \\
\text { actividades como uno más. Sen- } \\
\text { tirse miembro de la sociedad, } \\
\text { sentirse integrado y contar con } \\
\text { el apoyo de otros individuos. } \\
\text { - Derechos: ser considerado } \\
\text { igual que el resto de la gente, } \\
\text { que le traten con equidad, que } \\
\text { respeten su forma de ser, opi- } \\
\text { niones, deseos e intimidad }\end{array}$ & $\begin{array}{l}\text { Diez } \\
\text { residentes de } \\
\text { Matanzas, } \\
\text { Santa Cruz, } \\
\text { Tenerife } \\
\text { (España) }\end{array}$ & $\begin{array}{l}\text { Entrevista en profundi- } \\
\text { dad mediante guion }\end{array}$ & $\begin{array}{l}\text { La percepción de la calidad de vida } \\
\text { está por encima de la media, ya } \\
\text { que los entrevistados manifiestan } \\
\text { estar satisfechos con los servicios, } \\
\text { oportunidades y capacidades corres- } \\
\text { pondientes. Empero, es menester } \\
\text { realizar intervenciones desde el } \\
\text { trabajo social para la promoción de } \\
\text { la calidad de vida por medio de la } \\
\text { mediación de conflictos entre los ac- } \\
\text { tores. En ese sentido, ellos declaran } \\
\text { vivir en una paz pública, pero esta } \\
\text { puede verse amenazada por diferen- } \\
\text { cias entre comunidades al momento } \\
\text { de establecer la distribución de los } \\
\text { recursos }\end{array}$ \\
\hline 2010 & $\begin{array}{l}\text { Machado, } \\
\text { Anarte y Ruiz }\end{array}$ & $\begin{array}{l}\text { Calidad de vida: "la percepción } \\
\text { del individuo de su posición } \\
\text { en la vida en el contexto de la } \\
\text { cultura y sistema de valores en } \\
\text { los que vive y en relación con } \\
\text { sus objetivos, expectativas, } \\
\text { estándares y preocupaciones" } \\
\text { (Machado et al., } 2010 \text { p. 36) }\end{array}$ & 46 pacientes & Diabetes Quality of Life & $\begin{array}{l}\text { La ansiedad determinó la calidad } \\
\text { de vida }(\beta=0,595, t=3,321, p= \\
0,000) \text { al momento de interactuar } \\
\text { con otras cinco variables físicas, } \\
\text { médicas, psicológicas y sociales. La } \\
\text { depresión fue el segundo predictor } \\
\text { de la calidad de vida al interrelacio- } \\
\text { narse con otras cinco variables ( } \beta= \\
0,525, t=3,321, p=0,002)\end{array}$ \\
\hline 2010 & Grimaldo & $\begin{array}{l}\text { Calidad de vida: "estado de } \\
\text { satisfacción general, derivada de } \\
\text { la realización de potencialidades } \\
\text { de la persona. Posee aspectos } \\
\text { subjetivos y objetivos, la intimi- } \\
\text { dación, la expresión emocional, } \\
\text { la seguridad percibida, la pro- } \\
\text { ductividad personal y la salud; } \\
\text { y como aspectos objetivos se } \\
\text { consideran: el bienestar material, } \\
\text { las relaciones armónicas con el } \\
\text { ambiente físico y social y con la } \\
\text { comunidad objetivamente perci- } \\
\text { bida" (Grimaldo, 2010, p. 2) }\end{array}$ & $\begin{array}{l}198 \text { participan- } \\
\text { tes }\end{array}$ & $\begin{array}{l}\text { Escala de calidad de } \\
\text { vida }\end{array}$ & $\begin{array}{l}\text { Se estableció la validez y confiabi- } \\
\text { lidad de la calidad de vida conside- } \\
\text { rando nueve dimensiones relativas a } \\
\text { medios de comunicación }(\alpha=0,93) \text {, } \\
\text { bienestar económico }(\alpha=0,83) \text {, } \\
\text { pareja }(\alpha=0,87) \text {, vida familiar y } \\
\text { hogar }(\alpha=0,89) \text {, religión }(\alpha=0,95) \text {, } \\
\text { vecindario y comunidad }(\alpha=0,90) \text {, } \\
\text { ocio }(\alpha=0,88) \text {, amigos }(\alpha=0,88) \text { y } \\
\text { salud }(\alpha=0,85)\end{array}$ \\
\hline
\end{tabular}


184 Cruz García Lirios - JaVier Carreón Guillén - Jorge Hernández Valdés - Bertha Leticia Rivera Varela María Beatriz Castillo Escamilla - Erle García Estrada - José Francisco Rosas Ferrusca

\begin{tabular}{|c|c|c|c|c|c|}
\hline Año & Autores & Constructo & Muestra & Instrumentos & Resultados \\
\hline 2012 & Sadeghzadeh & $\begin{array}{l}\text { Quality life: "is a virtual } \\
\text { perception and containst the } \\
\text { negative and positive aspects of } \\
\text { the person's life and it explains } \\
\text { the way that a person perceived } \\
\text { the others aspects of his/her life } \\
\text { and how he/she reacts to them" } \\
\text { (Sadeghzadeh, 2012, p. 395) }\end{array}$ & 60 pacientes & $\begin{array}{l}\text { Medición pre y posinter- } \\
\text { vención }\end{array}$ & $\begin{array}{l}\text { Se establecieron diferencias signifi- } \\
\text { cativas entre los grupos experi- } \\
\text { mental y control con respecto a la } \\
\text { intervención médica y su calidad de } \\
\text { vida percibida antes y después de } \\
\text { la intervención ( } \mathrm{t}=3,86[29 \mathrm{gl}] \text {, } \mathrm{p} \\
=0,000)\end{array}$ \\
\hline 2012 & Derya & $\begin{array}{l}\text { Quality of life: "refers to } \\
\text { the impact of the workplace } \\
\text { satisfaction in non-work } \\
\text { life domains, in satisfaction } \\
\text { and reward expectations are } \\
\text { influenced by their work } \\
\text { environment employees jobs, } \\
\text { supervisors, and work groups, } \\
\text { and the organizational structure } \\
\text { and technology) and the extend } \\
\text { to which it provides value } \\
\text { rewards" (Derya, 2012,p. 196) }\end{array}$ & $\begin{array}{l}1.200 \text { emplea- } \\
\text { dos de hoteles } \\
\text { cinco estrellas }\end{array}$ & Quality of Worklife & $\begin{array}{l}\text { Los autores fijaron diferencias sig- } \\
\text { nificativas entre hombres y mujeres } \\
\text { respecto a su salud percibida }(\mathrm{t}= \\
2,543, \mathrm{p}=0,011) \text {, necesidades de } \\
\text { actualización }(\mathrm{t}=3,744, \mathrm{p}=0,000) \text {, } \\
\text { necesidades de conocimiento }(\mathrm{t}= \\
2,977, \mathrm{p}=0,003) \text { y necesidades } \\
\text { estéticas }(\mathrm{t}=1,790, \mathrm{p}=0,074)\end{array}$ \\
\hline 2012 & Tariq & $\begin{array}{l}\text { Life satisfaction: "refers to a } \\
\text { judgmental process in which } \\
\text { individual assess the quality } \\
\text { of their lives on the basis of } \\
\text { their own set of criteria" (Tariq, } \\
\text { 2012, p. 141) }\end{array}$ & $\begin{array}{l}\text { Cien ejecuti- } \\
\text { vos financieros }\end{array}$ & $\begin{array}{l}\text { Life Satisfaction and } \\
\text { Financial Stress }\end{array}$ & $\begin{array}{l}\text { La satisfacción de vida y el estrés } \\
\text { financiero correlacionaron negati- } \\
\text { vamente }(\mathrm{r}=-0118, \mathrm{p}<0,001) \text { y se } \\
\text { determinaron diferencias significati- } \\
\text { vas entre alto y bajo estrés financie- } \\
\text { ro con respecto a la satisfacción de } \\
\text { vida }(\mathrm{t}=2,37[98 \mathrm{gl}], \mathrm{p}<0,05)\end{array}$ \\
\hline 2013 & $\begin{array}{l}\text { Aristegui y } \\
\text { Vázquez }\end{array}$ & $\begin{array}{l}\text { Calidad de vida: "percepción } \\
\text { que los sujetos tienen sobre su } \\
\text { posición en la vida, su contexto } \\
\text { cultural y el sistema de valores } \\
\text { en el cual [viven], en relación } \\
\text { [con] sus expectativas, intereses } \\
\text { y logros" (Aristegui y Vázquez, } \\
\text { 2013,p. 7) }\end{array}$ & $\begin{array}{l}\text { Diez } \\
\text { transgéneros } \\
\text { argentinos }\end{array}$ & $\begin{array}{l}\text { Entrevistas en profun- } \\
\text { didad }\end{array}$ & $\begin{array}{l}\text { La calidad de vida y el bienestar } \\
\text { subjetivo o psicológico fueron } \\
\text { evocados en los discursos de los } \\
\text { entrevistados, ya que por una parte } \\
\text { declaran que sus expectativas de } \\
\text { vida son inhibidas al ser discrimina- } \\
\text { dos, pero por otra la formación de } \\
\text { un grupo transgénero les permitió } \\
\text { establecer redes de apoyo }\end{array}$ \\
\hline 2013 & $\begin{array}{l}\text { Abolfotouh, } \\
\text { Salam, } \\
\text { Alturaif } \\
\text {, Suliman, Al- } \\
\text { Essa, Al-Issa y } \\
\text { Al-Rowaily }\end{array}$ & $\begin{array}{l}\text { Quality of life: "which is } \\
\text { defined as a multidimensional } \\
\text { concept that encompasses the } \\
\text { physical, emotional, social } \\
\text { perception associated with an } \\
\text { illness or is treatment" (Abolfo- } \\
\text { tou et al., 2013, p. 1361) }\end{array}$ & 394 sujetos & Quality of Life & $\begin{array}{l}\text { Se dieron a conocer diferencias sig- } \\
\text { nificativas entre sexos con respecto } \\
\text { a su percepción de calidad de vida } \\
\left(X^{2}=13,622, p=0,001, t=0,316\right. \\
\mathrm{p}=0,001) \text { y satisfacción de vida } \\
\left(X^{2}=12,164, p=0,002, t=3,05, p\right. \\
=0,001)\end{array}$ \\
\hline
\end{tabular}




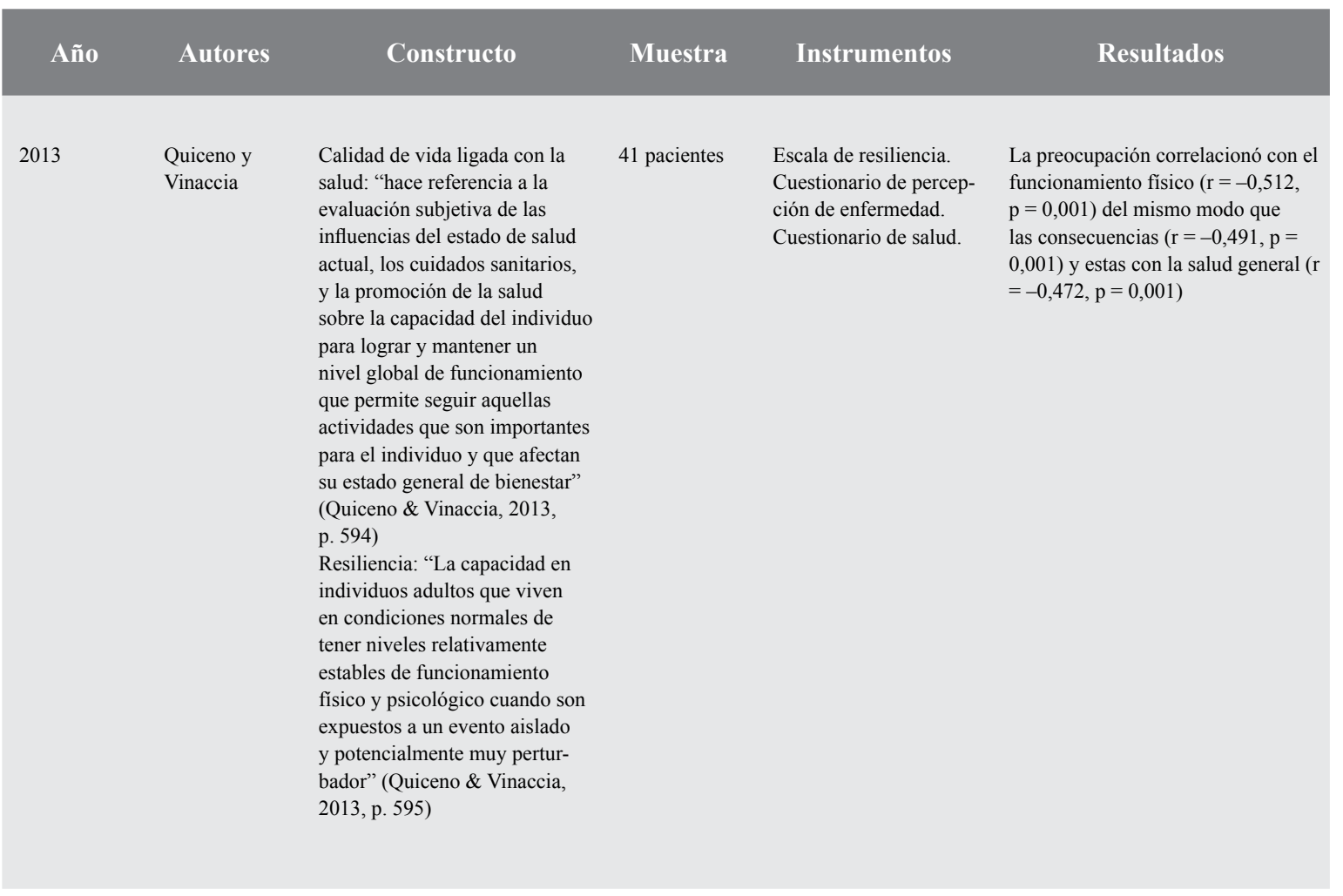

Fuente: elaboración propia.

La calidad de vida en su fase perceptual genera emociones de desconfianza hacia las autoridades que derivan en acciones ciudadanas disidentes (Carreón \& García, 2013). Antes bien, la percepción de calidad de vida, en cuanto a la noción de justicia social, se enlaza con estilos convencionales de desarrollo que el individuo ha aprendido desde su infancia y ahora en su etapa adulta traduce como fiabilidad o confianza, pero al darse cuenta de que el vínculo con sus autoridades es asimétrico, entonces moviliza los recursos necesarios para la desobediencia civil (García, 2013).

Tariq (2012) observa que la calidad de vida, en su dimensión de satisfacción vital, requiere de un conjunto de indicadores que oriente no solo la percepción del sujeto, sino además la acción colectiva. Un bajo nivel de satisfacción de vida es suficiente para activar el proceso de disidencia social, pero un alto nivel de satisfacción vital no crea nexos colaborativos, solidarios o empáticos (Carreón \& García, 2013).

Sin embargo, bajos niveles de satisfacción vital, los cuales sugieren mínimos estándares de calidad de vida, favorecen la formación de redes de apoyo. Es el caso de los nuevos movimientos sociales lésbico-gais o ecologistas, quienes al conformar grupos de autoayuda ocasionan un bienestar subjetivo mayor a quienes solo perciben abundancia de recursos (Carreón et al., 2013).

A medida que la calidad de vida se específica y delimita a factores psicológicos, se incrementan las expectativas de inconformidad, indignación y desobediencia civil, pero también afloran habilidades sociales como la creatividad e innovación de grupos minoritarios frente a la imposición ideológica o pragmática de las mayorías (Abolfotouh et al., 2013). 
En síntesis, la calidad de vida en términos económicos, políticos, sociales, sanitarios, educativos, laborales y tecnológicos es un constructo multidimensional (Quiceno \& Vinaccia, 2013).

\section{Especificación de un modelo de las variables correlacionadas con la calidad de vida}

Las relaciones entre oportunidades, capacidades, responsabilidades, justicia, fiabilidad y movilización dirigidas a la satisfacción vital pueden concretarse en un modelo (véase figura 1).

Se trata de 17 variables en torno a las cuales los vínculos de dependencia entre factores económicos, políticos, sociales, grupales y personales influyen en la satisfacción de vida.

La teoría del capital humano advierte que las capacidades tendrían un impacto significativo sobre la satisfacción vital, ya que un alto nivel de educación se compensa con un elevado estilo de vida satisfactorio (hipótesis 1).

Más aún, si la conexión entre capacidades y satisfacción parte de oportunidades reales o simbólicas, entonces como lo señala la teoría, el desarrollo humano está complementado (hipótesis 2).

Sin embargo, el proceso que supone libertades de elección, capacidades y satisfacción vital necesariamente, de acuerdo con la teoría de las demandas y recursos, se sujeta a la disponibilidad de recursos y su distribución entre las especies. En el caso de los recursos comunes existe una tragedia de suma cero en la que los actores involucrados en lugar de cooperar y establecer una corresponsabilidad administrativa de los recursos, compiten desenfrenadamente y suprimen la posibilidad de crecimiento de otras especies. Es por ello que la disponibilidad de recursos afecta de modo indirecto a la satisfacción vital (hipótesis 3).
No obstante que la disponibilidad de recursos marca la pauta en la organización de los grupos humanos, es el Estado en referencia a la ciudadanía, según lo advierte la teoría de la elección racional, quien incentiva la diseminación de los recursos. Cuando la relación entre sociedad civil y sus autoridades es asimétrica, entonces la calidad de vida se desvanece, pero cuando existen vínculos de confianza, entonces aflora la satisfacción vital (hipótesis 4).

En otro escenario, el nexo entre ciudadanía $\mathrm{y}$ autoridades genera expectativas de justicia que merman o enaltecen la corresponsabilidad. Esto es así porque, según la teoría ecológica del desarrollo, la política es concomitante con la economía. Mayores niveles de gobernabilidad se observan en países prósperos, mientras que la ingobernabilidad subyace en economías débiles. Es por ello que la justicia esperada incide directamente en la satisfacción vital de la ciudadanía para con su sistema político (hipótesis 5).

El proceso que parte de la confianza, capacidades y satisfacción vital es planteado por la teoría de la autosuficiencia (SFT). La relación entre sociedad y Estado orientada al desarrollo tiene en la formación del capital humano su vínculo principal. La SFT propone que el desarrollo, a diferencia del crecimiento, supone elevados estándares de satisfacción vital razón por la cual, las capacidades son un factor esencial en el nexo entre fiabilidad política y satisfacción de vida personal (hipótesis 6).

La relación indirecta entre justicia y satisfacción al ser mediada por las capacidades supone escenarios ecológicos de desarrollo. La teoría de los recursos de cambio (CRT) advierte que la conexión entre autoridades y ciudadanos se define por acciones molares y moleculares que afectarán la satisfacción individual.

Los actos molares, al contrario de las acciones moleculares, implican vínculos signifi- 
cativos que contrasta el individuo en situaciones de crisis. Por su parte, las relaciones poco significativas se entienden como actos moleculares desde los cuales no es posible construir una memoria colectiva, disidencia social o desobediencia civil.

Es así como la justicia, al considerarse un sistema de acciones molares, influye en la satisfacción vital mientras es regulada por las capacidades personales (hipótesis 7).

Sin embargo, la CRT sugiere que son las oportunidades las que inciden en las relaciones entre fiabilidad, justicia, capacidades y satisfacción. De este modo, las libertades de elección al vincularse con la fiabilidad política y capacidades individuales impactan en la satisfacción vital. Esto es así porque la confianza entre ciudadanos y políticos depende de opciones de elección en materia de empleo. Si existen oportunidades laborales, entonces la fiabilidad política se incrementa y al afectar las habilidades propicia altos estados de satisfacción (hipótesis 8). O bien, las oportunidades son diseminadas como factores de justicia incidiendo en la generación de habilidades laborales, mientras que es posible observar el acrecentamiento de satisfacción personal de vida (hipótesis 9).

Figura 1.

Especificación de relaciones

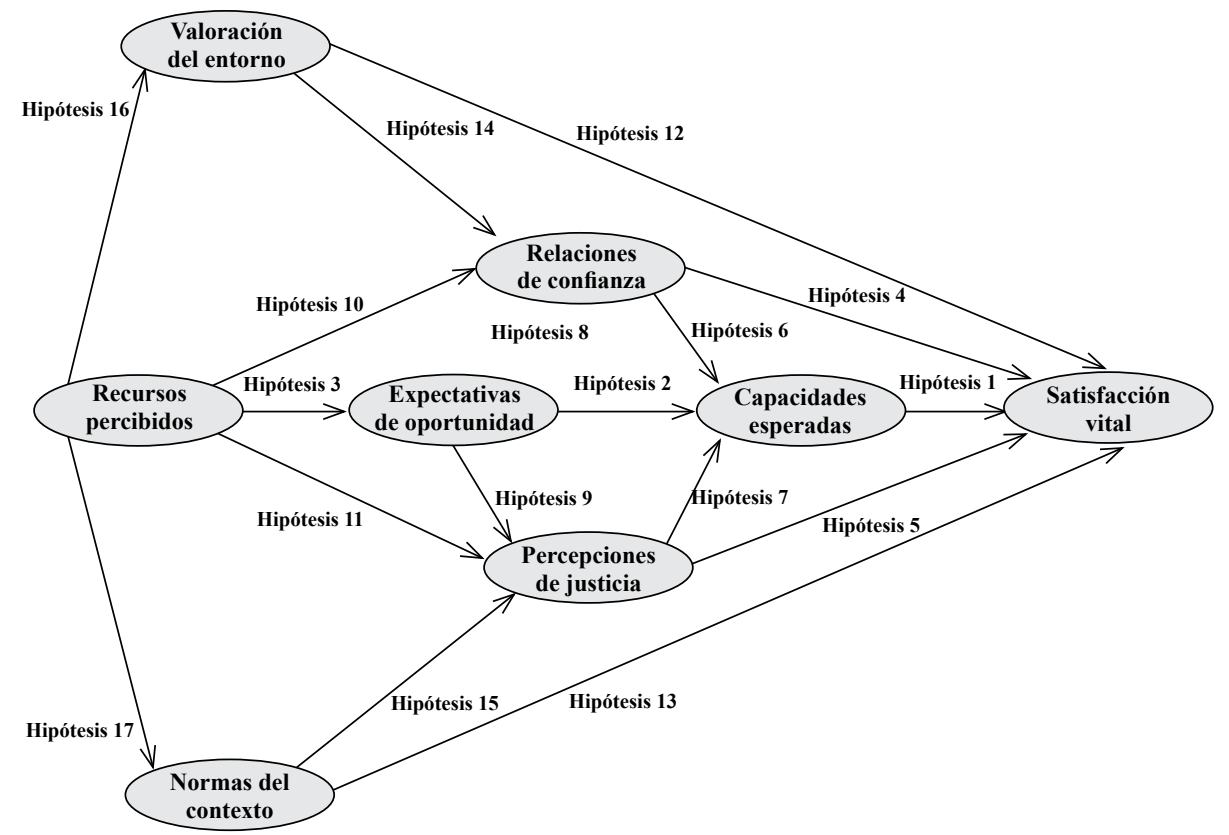

Fuente: elaboración propia.

Ahora bien, las libertades de elección implican decisiones, demandas y recursos a partir de los cuales las oportunidades se gestan. Es por esta cuestión que la CRT sostiene que la disponibilidad de recursos, pero sobre todo, el dilema de la distribución de los mismos, impac- ta en el desarrollo de comunidades o grupos que comparten escenarios y contextos.

La historia de una comunidad o grupo parece estar socavada por las redes de cooperación y confianza mientras la disponibilidad de 
recursos lo permita. Ante una situación de escasez emergen los dilemas y con ello las tragedias de las entidades comunes. En este sentido, la CRT afirma que las oportunidades son el resultado de una corresponsabilidad entre las partes implicadas.

De este modo, la gestión de recursos afecta indirectamente a la satisfacción a través de las oportunidades, fiabilidad, justicia y capacidades. En primera instancia, la carencia de recursos crearía una reducción de los niveles de confianza ciudadana ante la administración pública, ello impactaría en las opciones de elección aminorando la capacidad de consumo. Una vez que el poder adquisitivo está comprometido, los niveles de satisfacción ciudadana se reducen a su mínima expresión. En este tenor, la escasez también puede incidir en percepciones de injusticia ciudadana, frente a la falta de atingencia gubernamental, las capacidades de elección se reducen hasta influir en la satisfacción vital (hipótesis 10).

En contraste, cuando la disponibilidad de recursos es insuficiente pero ampliamente percibida por la ciudadanía, las oportunidades de elección parecen actuar en la confianza ciudadana y con ello en la toma de decisiones buscando la satisfacción no solo personal o grupal, sino social. Esto también supone un proceso de justicia ante la distribución de recursos vía los servicios públicos. En este escenario, la satisfacción vital precede a las opciones de elección que al percibirse como abundantes transfieren el efecto de la justicia social hacia la satisfacción vital (hipótesis 11).

Así mismo, la relación entre la valoración del entorno (recursos disponibles) y la satisfacción de vida (necesidades obtenidas) la entiende la CRT como un proceso directo y significativo (hipótesis 12). Se trata de una relación asimétrica, ya que la escasez de recursos frente al aumento de expectativas suscita un escenario que compromete la confianza de la ciudadanía para con sus futuros gobernantes y las capacidades de la sociedad civil futura (hipótesis 14 y 16).

La CRT advierte que en el caso de las normas sociales en cuanto a la administración y, por ende, la distribución de los recursos, su vinculación con la satisfacción al ser directa y significativa comprende un reduccionismo estructural (hipótesis 13), o bien, en la interrelación con la justicia social y las capacidades implica la emergencia de un sistema democrático de coparticipación ciudadana frente a la apertura del Estado (hipótesis 15 y 17).

En resumen, la especificación de relaciones entre los factores que las teorías emplean para explicar la incidencia de la disponibilidad de recursos sobre la satisfacción vital conlleva:

- La formación de capacidades entendidas como opciones de elección, orienta la conformación de capital humano como factor de desarrollo cuando menos económico.

- Oportunidades, capacidades y responsabilidades percibidas no desde la disponibilidad física de los recursos, sino desde las expectativas de consumo que los servicios públicos diseminan en los usuarios.

- Debido a que los recursos son administrados en primera instancia por el Estado, generan percepciones de confianza que enaltecen las opciones de elección, pero la desconfianza inhibe las alternativas de elección y con ello la satisfacción vital.

- La disponibilidad común de los recursos desencadena relaciones de dependencia entre los factores esgrimidos, con el objetivo de incentivar las oportunidades de elección y con ello alcanzar la satisfacción vital.

En consecuencia: Los vínculos de dependencia que se concretan en el modelo se ajustan a los datos observados. Es decir, la percepción de disponibilidad y comunalidad de los recursos impacta directa e indirectamente en la satisfacción de vida de los estudiantes. En el segundo caso de relación indirecta, las expectativas de oportunidad, confianza, justicia y capacidad al reducir o aumentar la incidencia 
de los recursos esperados sobre la satisfacción vital, ajustan sus relaciones especificadas a los datos obtenidos.

\section{Método}

Se llevó a cabo un estudio transversal, realizando una selección no probabilística de 245 estudiantes. El criterio de elección fue el contar con una actividad remunerada, servicio de Internet y haber sido inscrito en el periodo lectivo escolar. Se entrevistaron a 120 mujeres y 125 hombres $(M=20,13$ años de edad y DE $=2,36$ años de edad). El estatus económico al que pertenece la muestra fue medio bajo con alrededor de 1.500 USD mensuales de ingreso familiar ( $\mathrm{M}=950 \mathrm{USD}$ y $\mathrm{DE}=24,5 \mathrm{USD})$.

Respecto a la educación los participantes declararon pertenecer a la universidad pública $(\mathrm{M}=2,13$ años de estudio y $\mathrm{DE}=0,47$ años de estudio). El $67 \%$ de los entrevistados contaba con una beca o apoyo económico $(\mathrm{M}=100$ USD y DE $=7,5$ USD). Los gastos relativos a colegiatura (300 USD semestral), útiles (250 USD semestral), Internet (50 USD mensual) y transporte (35 USD semanal) generan una inversión aproximada de 1.000 USD semestral $(\mathrm{M}=870 \mathrm{USD}$ y $\mathrm{DE}=15,67 \mathrm{USD})$.
En el tema laboral el $36 \%$ de la muestra declaró trabajar antes o después de asistir al colegio ( $\mathrm{M}=400 \mathrm{USD}$ mensual y $\mathrm{DE}=23,5 \mathrm{USD}$ mensual). De los entrevistados que laboran el $78 \%$ señala que su salario se incrementaría sustancialmente si concluyera su formación profesional $(\mathrm{M}=1.200 \mathrm{USD}$ mensuales esperados y $\mathrm{DE}=45,5$ USD mensuales esperados). Por el contrario, el $84 \%$ de los entrevistados declaró que sus ingresos serían inferiores si solo contaran con la formación media básica $(\mathrm{M}=250$ USD y $\mathrm{DE}=14,6 \mathrm{USD})$.

En relación a la tecnología, el 57 \% paga mensualmente su acceso a Internet desde una línea fija ( $\mathrm{M}=470$ USD mensuales y $\mathrm{DE}=$ 15,7 USD mensuales) mientras que el $93 \%$ cuenta con servicio de telefonía móvil $(\mathrm{M}=140$ USD mensuales y $\mathrm{DE}=10,2$ USD mensuales). Facebook (46\%), Twitter (27\%) y Google+ $(14 \%)$ son las redes sociales de mayor uso para fines escolares o búsqueda de empleo.

Se construyó un cuestionario de calidad de vida a partir de las dimensiones educativa, tecnológica y laboral en función de los factores de disponibilidad de recursos, fiabilidad social, justicia social, oportunidades de elección, habilidades de selección y percepciones de satisfacción (véase tabla 2).

Tabla 2.

Operacionalización de variables

\begin{tabular}{|c|c|c|c|}
\hline Factor & Definición & Ítems & Ponderación \\
\hline $\begin{array}{l}\text { Satisfacción } \\
\text { vital }\end{array}$ & $\begin{array}{l}\text { Grado de evaluación de los servicios públicos } \\
\text { (educación, tecnología y empleo) }\end{array}$ & $\mathrm{SV} 1, \mathrm{SV} 2, \mathrm{SV} 3$ & $\begin{array}{l}0=\text { nada satisfactorio, } 1=\text { muy poco satisfactorio, } 2=\text { poco } \\
\text { satisfactorio, } 3=\text { muy satisfactorio }\end{array}$ \\
\hline $\begin{array}{l}\text { Capacidades } \\
\text { esperadas }\end{array}$ & $\begin{array}{l}\text { Nivel de habilidades de elección educativa, } \\
\text { tecnológica y laboral }\end{array}$ & CE1, CE2, CE3 & $\begin{array}{l}0=\text { nada preferible, } 1=\text { muy poco preferible, } 2=\text { poco } \\
\text { preferible, } 3=\text { muy preferible }\end{array}$ \\
\hline $\begin{array}{l}\text { Relaciones } \\
\text { de confianza }\end{array}$ & $\begin{array}{l}\text { Grado de credibilidad en las autoridades respecto } \\
\text { a educación, tecnología y empleo }\end{array}$ & $\mathrm{RC} 1, \mathrm{RC} 2, \mathrm{RC} 3$ & $\begin{array}{l}0=\text { nada fiable, } 1=\text { muy poco fiable, } 2=\text { poco fiable, } 3=\text { muy } \\
\text { fiable }\end{array}$ \\
\hline $\begin{array}{l}\text { Percepción } \\
\text { de justicia }\end{array}$ & $\begin{array}{l}\text { Nivel de evaluación de la administración pública } \\
\text { en materia educativa, tecnológica y laboral }\end{array}$ & PJ1, PJ2, PJ3 & $\begin{array}{l}0=\text { nada deseable }, 1=\text { muy poco deseable }, 2=\text { poco deseable } \\
3=\text { muy deseable }\end{array}$ \\
\hline $\begin{array}{l}\text { Expectativas } \\
\text { de oportunidad }\end{array}$ & $\begin{array}{l}\text { Grado de libertad de elección educativa, tecno- } \\
\text { lógica y laboral }\end{array}$ & EO1, EO2, EO3 & $\begin{array}{l}0=\text { nada opcional, } 1=\text { muy poco opcional, } 2=\text { poco opcional } \\
3=\text { muy opcional }\end{array}$ \\
\hline $\begin{array}{l}\text { Valoración } \\
\text { del entorno }\end{array}$ & $\begin{array}{l}\text { Nivel de acceso a servicios públicos educativos, } \\
\text { tecnológicos y laborales }\end{array}$ & VE1, VE2, VE3 & $\begin{array}{l}0=\text { nada eficiente, } 1=\text { muy poco eficiente, } 2=\text { poco eficiente, } 3 \\
=\text { muy eficiente }\end{array}$ \\
\hline $\begin{array}{l}\text { Normas } \\
\text { de contexto }\end{array}$ & $\begin{array}{l}\text { Grado de distribución de los servicios públicos } \\
\text { educativos, tecnológicos y laborales }\end{array}$ & $\mathrm{NC} 1, \mathrm{NC} 2, \mathrm{NC} 3$ & $\begin{array}{l}0=\text { nada apreciable }, 1=\text { muy poco apreciable }, 2=\text { poco } \\
\text { apreciable }, 3=\text { muy apreciable }\end{array}$ \\
\hline $\begin{array}{l}\text { Recursos } \\
\text { percibidos }\end{array}$ & $\begin{array}{l}\text { Nivel de disponibilidad de recursos a través de } \\
\text { servicios educativos, tecnológicos y laborales }\end{array}$ & $\mathrm{RP} 1, \mathrm{RP} 2, \mathrm{RP} 3$ & $\begin{array}{l}0=\text { nada cooperativo, } 1=\text { muy poco cooperativo, } 2=\text { poco } \\
\text { cooperativo, } 3=\text { muy cooperativo }\end{array}$ \\
\hline
\end{tabular}

Fuente: elaboración propia. 
190 Cruz García Lirios - JaVier Carreón Guillén - Jorge Hernández Valdés - Bertha Leticia Rivera Varela María Beatriz Castillo Escamilla - Erle García Estrada - José Francisco Rosas Ferrusca

La aplicación de las encuestas se llevó a cabo en las instalaciones de la Universidad Autónoma del Estado de México, previa tramitación ante las autoridades de la institución. Al momento de resolver el cuestionario, los entrevistados fueron instruidos para escribir las dudas que pudieran tener respecto a la encuesta. Una vez se recopiló la información se capturó en el paquete estadístico para ciencias sociales (SPSS, por su sigla en inglés) versión 21,0, a fin de estimar los parámetros multivariables en el análisis de momentos estructurales (Amos por su acrónimo en inglés) versión 6,0 .

Se realizó un análisis de normalidad considerando el parámetro de curtosis así como de confiabilidad asumiendo una consistencia interna o alfa de Cronbach. Posteriormente se procedió a estimar la validez de constructo previa esfericidad y adecuación del instrumento a la muestra de estudio. Enseguida, se estimaron correlaciones bivariadas o covarianzas multivariables para anticipar relaciones causales en modelos estructurales, ajuste y residuos.

Normalidad. Se utilizó el parámetro de curtosis para determinar la proximidad de las respuestas a la media y desviación estándar. Los valores cercanos a la unidad se asumieron como evidencias de distribución normal.

Confiabilidad. La consistencia interna de los reactivos con respecto a la escala se ponderó con el estadístico alfa de Cronbach. Los valores superiores a 0,60 y menores a 0,90 fueron prueba de relaciones simétricas entre los reactivos y los rasgos psicológicos que se pretendieron medir.

Validez. Se ponderó la adecuación con el parámetro Kaiser-Meyer-Olkin en el que los valores superiores a 6,00 se consideraron un requerimiento para la validez de constructo. También se estimó la esfericidad con la prueba de Bartlett, el valor Chi-cuadrado cercano a la unidad y nivel de significancia menor a 0,050 se tomaron como un segundo requerimiento para el análisis fac- torial exploratorio de componentes principales con rotación varimax. Las correlaciones entre el ítem y el factor superiores a 0,300 se asumieron como indicadores de un constructo. En cuanto a los porcentajes de varianza explicada superiores a $20 \%$ se estimaron como prueba de ajuste de las especificaciones del modelo con respecto a los datos en observación.

Correlación. Se empleó el parámetro $r$ de Pearson para calcular las relaciones negativas o positivas entre los factores establecidos en la validez de constructo. Aquellos valores cercanos a cero y la unidad se admitieron como relaciones espurias y colineales. En cambio, los valores entre los extremos fueron considerados como asociaciones probables de dependencia.

Covarianza. Se utilizó la estimación phi para determinar la asociación entre un factor y otro en referencia a los demás factores. Al igual que la correlación, los valores se identificaron como relaciones espurias, colineales y de dependencia.

Estructura. Se usaron parámetros phi para estimar covarianzas, estadísticos gamma para la regresión de factores exógenos a mediadores y parámetros beta para la regresión de factores mediadores a endógenos. Los estadísticos delta, épsilon y sigma se emplearon para calcular los errores de medición de indicadores exógenos y endógenos así como el disturbio de factores endógenos. El criterio para interpretar los valores cercanos a cero y a la unidad también se valoró para los parámetros en mención.

Ajuste. El contraste del modelo se llevó a cabo a partir de la estimación del índice de bondad de ajuste (GFI, por su sigla en inglés). Los valores cercanos a la unidad se juzgaron como demostración de aceptación de hipótesis nula.

Residual. El contraste del modelo también se realizó desde el cálculo del error de aproximación cuadrático medio (RMSEA, por su sigla en inglés). Los valores cercanos a cero 
fueron considerados como evidencia de ajuste del modelo especificado con respecto a los datos obtenidos.

\section{Resultados}

Se establecieron ocho factores [KMO $\left.=6,25 ; \mathrm{X}^{2}=14,25(23 \mathrm{gl}) ; \mathrm{p}=0,000\right]$ (véase tabla 3 ) relativos a la satisfacción de vida (ítems SV1, SV2, SV3 y $45 \%$ de la varianza total explicada), capacidades esperadas (CE1, CE2,
CE3 y $37 \%$ de la varianza total explicada), relaciones de confianza (RC1, RC2, RC3 y $33 \%$ de la varianza explicada), percepción de justicia (PJ1, PJ2, PJ3 y $31 \%$ de la varianza explicada), expectativas de oportunidad (EO1, EO2, EO3 y $27 \%$ de la varianza explicada), valoraciones del entorno (VE1, VE2, VE3 y $25 \%$ de la varianza explicada), normas de contexto (NC1, NC2, NC3 y $23 \%$ de la varianza explicada) y recursos percibidos (RP1, RP2, RP3 y $21 \%$ de la varianza explicada).

Tabla 3.

Validez de los constructos

que se correlacionan con la calidad de vida

\begin{tabular}{|c|c|c|c|c|c|c|c|c|c|}
\hline & & F1 & F2 & F3 & F4 & F5 & F6 & F7 & F8 \\
\hline & Satisfacción de vida & & & & & & & & \\
\hline SV1 & De las cosas que me gusta hacer, estudiar me parece: & 0,356 & & & & & & & \\
\hline SV2 & De las tecnologías que me interesan, el Internet que utilizo es: & 0,451 & & & & & & & \\
\hline \multirow[t]{2}{*}{ SV3 } & De mis preferencias de empleo, la bolsa de trabajo universitaria es: & 0,478 & & & & & & & \\
\hline & Capacidades esperadas & & & & & & & & \\
\hline CE1 & De las cosas que puedo hacer, estudiar me parece: & & 0,591 & & & & & & \\
\hline CE2 & De las habilidades que tengo, usar el Internet para tareas es: & & 0,894 & & & & & & \\
\hline \multirow[t]{2}{*}{ CE3 } & De los trabajos que he tenido, recibir una beca es: & & 0,621 & & & & & & \\
\hline & Relaciones de confianza & & & & & & & & \\
\hline RC1 & De entre todas mis amistades, estudiar con alguien desconocido es: & & & 0,412 & & & & & \\
\hline RC2 & De entre todos mis contactos, mandar mi tarea a un correo de la universidad es: & & & 0,512 & & & & & \\
\hline \multirow[t]{2}{*}{ RC3 } & De mis trabajos anteriores, cualquier labor extracurricular me parece: & & & 0,725 & & & & & \\
\hline & Percepción de justicia & & & & & & & & \\
\hline PJ1 & De los rechazados en el examen de admisión, faltar a una clase me parece: & & & & 0,412 & & & & \\
\hline PJ2 & De las páginas electrónicas que nos espían, enviar mi tarea por la red universitaria es: & & & & 0,587 & & & & \\
\hline \multirow[t]{2}{*}{ PJ3 } & $\begin{array}{l}\text { De los empleos con salario mínimo, la posibilidad de ganar dinero en la universidad } \\
\text { es: }\end{array}$ & & & & 0,824 & & & & \\
\hline & Expectativas de oportunidad & & & & & & & & \\
\hline EO1 & De las oportunidades de trabajar que he tenido, recibir una beca me parece: & & & & & 0,472 & & & \\
\hline EO2 & $\begin{array}{l}\text { De las tecnologías más útiles, capacitarme en o con alguna de ellas en la universidad } \\
\text { es: }\end{array}$ & & & & & 0,384 & & & \\
\hline \multirow[t]{2}{*}{ EO3 } & $\begin{array}{l}\text { De los empleos que me han enseñado, la posibilidad de especializarme en la univer- } \\
\text { sidad es: }\end{array}$ & & & & & 0,481 & & & \\
\hline & Valoraciones del entorno & & & & & & & & \\
\hline VE1 & De los servicios públicos que he pagado, estudiar en universidad gratuita me parece: & & & & & & 0,412 & & \\
\hline VE2 & De las redes públicas de Internet que conozco, usar el servidor de la universidad es: & & & & & & 0,382 & & \\
\hline \multirow[t]{2}{*}{ VE3 } & $\begin{array}{l}\text { De los servicios de colocación de empleo en los que estoy inscrito, la bolsa universi- } \\
\text { taria es: }\end{array}$ & & & & & & 0,482 & & \\
\hline & Normas de contexto & & & & & & & & \\
\hline NC1 & De las cosas que acostumbro hacer, leer para un examen me parece: & & & & & & & 0,413 & \\
\hline NC2 & De los contactos con los que acostumbro platicar, leer sus comentarios es: & & & & & & & 0,482 & \\
\hline \multirow[t]{2}{*}{ NC3 } & De las actividades que acostumbro hacer, realizar trabajos finales es: & & & & & & & 0,583 & \\
\hline & Recursos percibidos & & & & & & & & \\
\hline RP1 & De las cosas que he compartido, prestar libros a compañeros de clase es: & & & & & & & & 0,351 \\
\hline $\mathbf{R P 2}$ & $\begin{array}{l}\text { De los mensajes que he enviado o recibido en Internet, darme de baja en la red me } \\
\text { parece: }\end{array}$ & & & & & & & & 0,486 \\
\hline RP3 & De las cosas que he hecho por dinero, hacer la tarea de alguien me parece: & & & & & & & & 0,631 \\
\hline
\end{tabular}

Fuente: elaboración propia. 
En el caso de los resultados descriptivos (véase tabla 4) se observa una tendencia a opciones positivas para las respuestas a ítems de los factores de satisfacción de vida, capacidades esperadas, relaciones de confianza, normas de contexto y recursos percibidos y una prevalencia negativa para los factores de percepción de justicia, expectativas de oportunidad y valoraciones del entorno.

En lo que atañe a la satisfacción de vida, el ítem SV3 ( $\mathrm{M}=2,47 ; \mathrm{DE}=0,49 ; \mathrm{C}=2,04)$ obtuvo el valor más cercano a la opción "muy satisfactorio" mientras que el ítem SV2 $(\mathrm{M}=$ 2,01; $\mathrm{DE}=0,27 ; \mathrm{C}=2,08$ ) se aproximó a la opción "poco satisfactorio". Es decir, la muestra encuestada parece orientar su satisfacción vital hacia un escenario educativo, tecnológico y laboral cercano a la satisfacción plena.

En el caso de las capacidades esperadas, el ítem CE3 ( $\mathrm{M}=2,71 ; \mathrm{DE}=0,41 ; \mathrm{C}=2,01)$ rondó la "muy preferible". En contraste, el ítem
CE1 $(\mathrm{M}=2,47 ; \mathrm{DE}=0,37 ; \mathrm{C}=2,01)$ se acercó a la opción "poco preferible". En este sentido, la muestra encuestada parece dirigir sus respuestas a una opción próxima a la percepción eficiente de sus capacidades.

Por su parte, en las relaciones de confianza, el ítem RC2 $(\mathrm{M}=2,94 ; \mathrm{DE}=0,48$; $\mathrm{C}=2,05)$, al contrario del ítem $\mathrm{RC} 3(\mathrm{M}=2,04$; $\mathrm{DE}=0,93 ; \mathrm{C}=2,01)$ se acercó a la opción "muy fiable". Ello sugiere que la confianza se percibe como un elemento fundamental de las relaciones entre la muestra encuestada.

En cuanto a la percepción de justicia, el ítem $\mathrm{PJ} 3(\mathrm{M}=1,27 ; \mathrm{DE}=0,31 ; \mathrm{C}=2,05)$ respecto al ítem PJ2 $(\mathrm{M}=1,59 ; \mathrm{DE}=0,15 ; \mathrm{C}=2,01)$ al estar rondando la alternativa "muy poco deseable" supone una apreciación sesgada de justicia. Esto es así porque la injusticia que la muestra encuestada percibe de sus autoridades parece ser un elemento central en su satisfacción de vida.

Tabla 4.

\section{Descriptivos del instrumento}

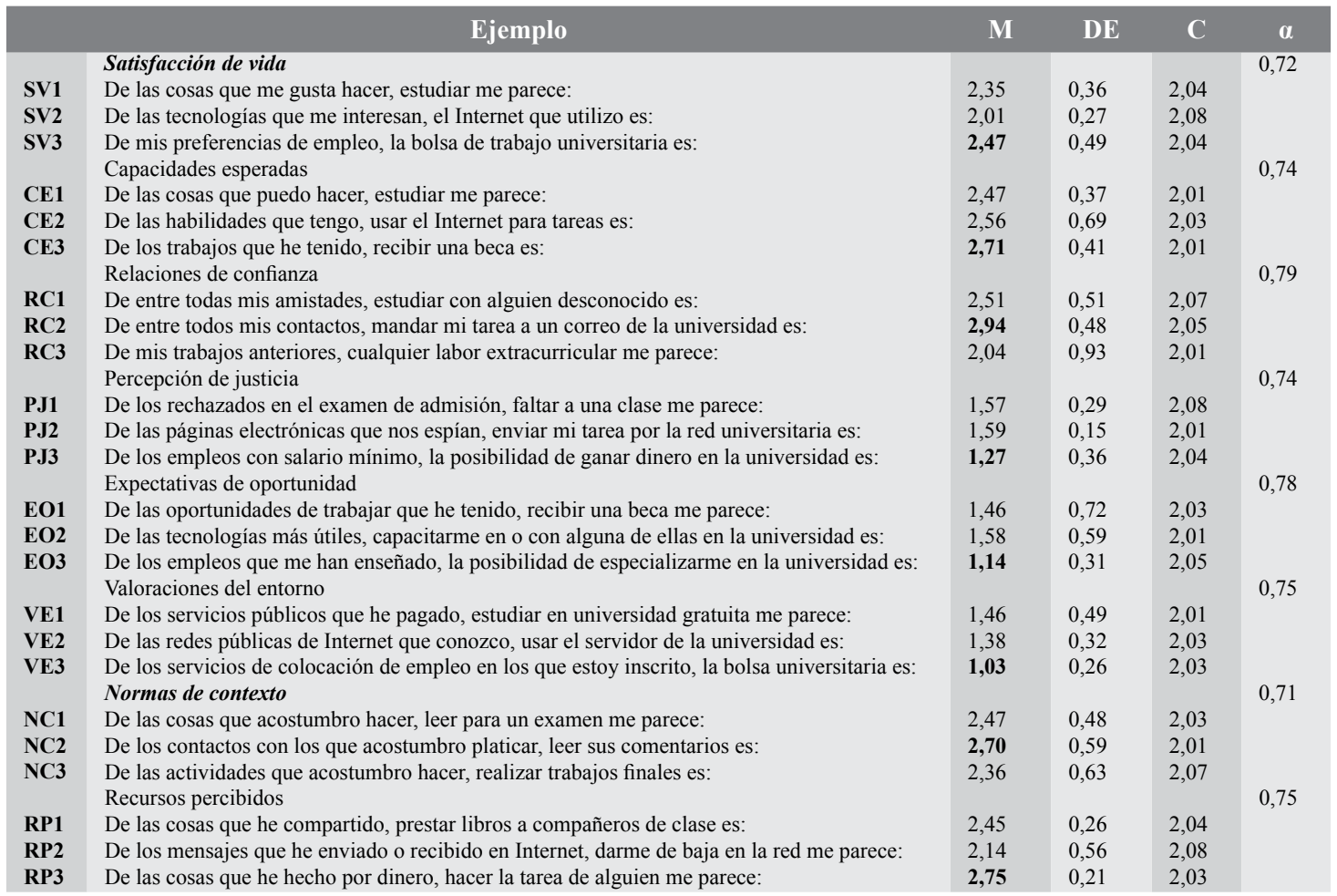

Fuente: elaboración propia. 
En el caso de las expectativas de oportunidad, el ítem EO3 $(\mathrm{M}=1,14 ; \mathrm{DE}=$ $0,31 ; \mathrm{C}=2,05)$ en referencia al ítem EO2 $(\mathrm{M}=$ $1,38 ; \mathrm{DE}=0,59 ; \mathrm{C}=2,01)$ refleja una tendencia a la opción "muy poco opcional", lo que hace suponer que las oportunidades se valoran como casi nulas por parte de la muestra encuestada.

Es el mismo caso de las valoraciones del entorno, ya que el ítem VE3 $(\mathrm{M}=1,03 ; \mathrm{DE}=$ $0,26 ; \mathrm{C}=2,03)$ respecto al ítem VE1 $(\mathrm{M}=1,46$; $\mathrm{DE}=0,49 ; \mathrm{C}=2,01)$ evidencia una inclinación a la opción "muy poco eficiente", que es el resultado de considerar a los servicios públicos como un sistema de distribución inequitativo entre la muestra encuestada.

En cuanto a las normas de contexto, el ítem NC2 $(\mathrm{M}=2,70 ; \mathrm{DE}=0,59 ; \mathrm{C}=2,01)$ en contraste con el ítem NC3 $(\mathrm{M}=2,35$; DE $=0,63 ; \mathrm{C}=2,07)$ se acercó a la opción "muy apreciable". Esto significa que las convenciones que guían las acciones del individuo al ser evaluadas positivamente prueban la influencia del sistema sobre un indicador de la calidad de vida en la muestra encuestada.

Por último, en el factor de los recursos percibidos, el ítem RP3 $(\mathrm{M}=2,75 ; \mathrm{DE}=$ $0,21 ; \mathrm{C}=2,03)$ en comparación con el ítem $\mathrm{RP} 2(\mathrm{M}=2,14 ; \mathrm{DE}=0,56 ; \mathrm{C}=2,08)$ revela una tendencia "muy cooperativa" por parte de la muestra encuestada. Ello quiere decir que la distribución de los recursos probablemente incide en la percepción de su escasez y, por ende, en la necesidad de compartirlos.

La confiabilidad de los factores 1 al 8 (alfas respectivas de 0,$72 ; 0,74 ; 0,79 ; 0,74 ; 0,78$; $0,75 ; 0,71$ y 0,75$)$ manifiesta una consistencia regular entre los ítems, ya que la confiabilidad general del instrumento fue de 0,69 .

La asociación entre los factores (véase tabla 5) muestra que la satisfacción de vida y las expectativas de oportunidad $(r=0,582)$, capacidades esperadas y recursos percibidos $(\mathrm{r}=0,719)$, relaciones de confianza y recursos percibidos $(r=0,625)$, percepción de justicia y expectativas de oportunidad $(0,613)$, expectativas de oportunidad y recursos percibidos $(0,509)$, valoración del entorno y normas de contexto $(0,495)$ y normas de contexto y recursos percibidos $(r=0,321)$ suponen que la especificación de relaciones entre los factores podría ser estimada por un modelo, aunque se esperaba que hubiesen relaciones negativas entre percepciones de justicia, expectativas de oportunidad y valoraciones del entorno, ya que sus medias, desviaciones y curtosis advertían una prevalencia negativa con respecto a la tendencia positiva de los demás factores.

Tabla 5.

Correlaciones entre los factores

\begin{tabular}{|c|c|c|c|c|c|c|c|c|}
\hline & SV & CA & $\mathbf{R C}$ & PJ & EO & VE & NC & $\mathbf{R P}$ \\
\hline Satisfacción de vida & 1,000 & & & & & & & \\
\hline Capacidades esperadas & 0,461 & 1,000 & & & & & & \\
\hline Relaciones de confianza & 0,372 & 0,592 & 1,000 & & & & & \\
\hline Percepciones de justicia & 0,461 & 0,491 & 0,381 & 1,000 & & & & \\
\hline Expectativas de oportunidad & 0,582 & 0,395 & 0,493 & 0,613 & 1,000 & & & \\
\hline Valoraciones del entorno & 0,482 & 0,591 & 0,597 & 0,492 & 0,315 & 1,000 & & \\
\hline Normas de contexto & 0,481 & 0,391 & 0,570 & 0,582 & 0,526 & 0,495 & 1,000 & \\
\hline Recursos percibidos & 0,493 & 0,719 & 0,625 & 0,481 & 0,593 & 0,314 & 0,321 & 1,000 \\
\hline
\end{tabular}

Fuente: elaboración propia. 
El análisis de covarianzas (véase tabla 6) al igual que el de las correlaciones, mostró vínculos positivos entre satisfacción de vida y valoraciones del entorno $(\Phi=0,610)$, capacidades esperadas y expectativas de oportunidad $(\Phi$ $=0,692)$, relaciones de confianza y recursos percibidos $(\Phi=0,729)$, percepción de justicia y recursos percibidos $(\Phi=0,624)$, expectativas de oportunidad y normas de contexto $(\Phi=0,714)$, valoraciones del entorno y recursos percibidos $(\Phi=0,624)$ y normas de contexto y recursos percibidos $(\Phi=0,735)$.

Tabla 6.

Covarianzas entre los factores

\begin{tabular}{|c|c|c|c|c|c|c|c|c|}
\hline & SV & $\mathbf{C A}$ & $\mathbf{R C}$ & $\mathbf{P J}$ & EO & $\mathbf{V E}$ & $\mathbf{N C}$ & $\mathbf{R P}$ \\
\hline Satisfacción de vida & 1,825 & & & & & & & \\
\hline Capacidades esperadas & 0,372 & 1,935 & & & & & & \\
\hline Relaciones de confianza & 0,482 & 0,481 & 1,725 & & & & & \\
\hline Percepciones de justicia & 0,592 & 0,593 & 0,371 & 1,868 & & & & \\
\hline Expectativas de oportunidad & 0,393 & 0,692 & 0,484 & 0,371 & 1,938 & & & \\
\hline Valoraciones del entorno & 0,610 & 0,482 & 0,596 & 0,415 & 0,687 & 1,836 & & \\
\hline Normas de contexto & 0,413 & 0,396 & 0,686 & 0,581 & $\mathbf{0 , 7 1 4}$ & 0,482 & 1,738 & \\
\hline Recursos percibidos & 0,382 & 0,481 & 0,729 & 0,624 & 0,491 & 0,624 & 0,735 & 1,829 \\
\hline
\end{tabular}

Fuente: elaboración propia.

Las covarianzas esgrimidas advierten que las relaciones especificadas parecen establecer un sistema de calidad de vida centrado en la satisfacción de vida, capacidades esperadas, relaciones de confianza, normas de contexto y recursos percibidos más que en percepciones de justicia, expectativas de oportunidad y valoraciones del entorno (véase Figura 2).

Figura 2.

Relaciones estructurales

entre las variables correlacionadas con la calidad de vida

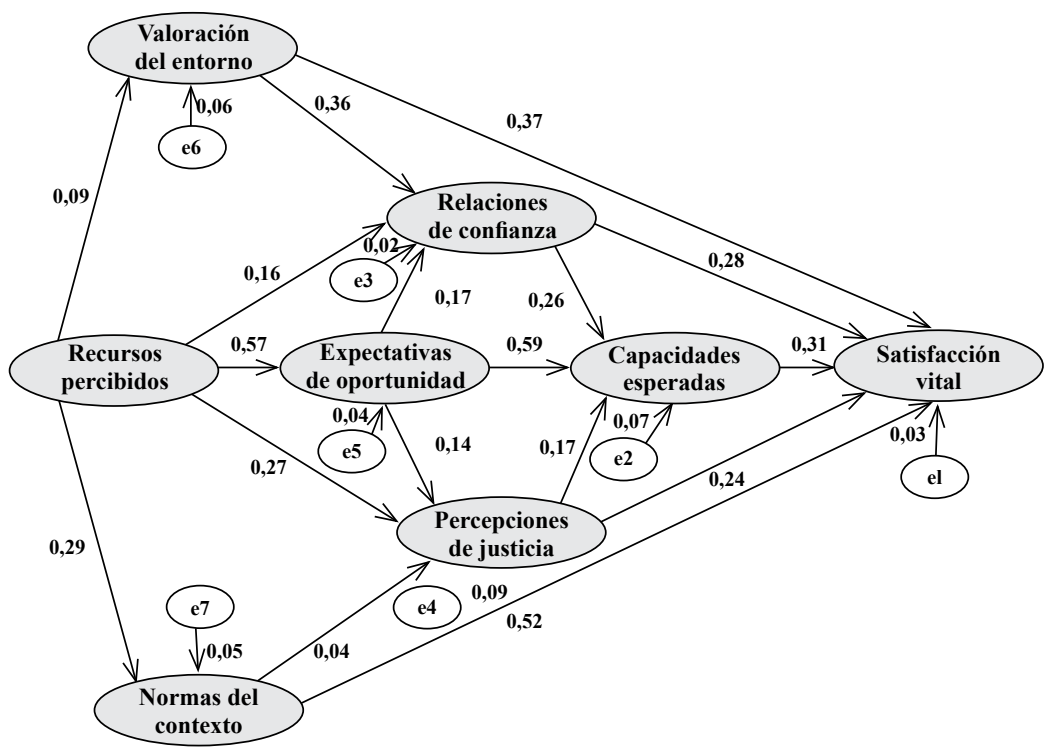

Fuente: elaboración propia. 
El modelo de relaciones estructurales muestra que el factor que influye en la percepción de los recursos sobre la satisfacción de vida es la norma de contexto $(\gamma=0,52)$, seguida del factor de valoración del entorno $(\gamma=0,37)$, capacidades esperadas $(\beta=0,31)$, relaciones de confianza $(\beta=0,28)$ y percepción de justicia $(\beta=$ $0,24)$. Es decir, la plena satisfacción de acciones relativas a la academia, tecnología y empleo está influida por la disponibilidad de los recursos percibidos a través de los comportamientos arraigados de los estudiantes. Este hallazgo enaltece un supuesto de la teoría de los recursos comunes según el cual, los usos y costumbres de los grupos internalizan los recursos como elementos de comunidad e identidad (García, Carreón \& Hernández, 2014). Esto es así porque la conservación de los recursos obedece a costumbres enraizadas en las que el sentido de pertenencia es fundamental para la satisfacción personal, grupal o comunal.

El contraste de las relaciones de dependencia $\left[\mathrm{X}^{2}=12,35\right.$ (12 gl); $\mathrm{p}=0,000$; GFI $=0,975 ; \mathrm{RMR}=0,000]$ evidencia la aceptación de la hipótesis nula.

\section{Discusión}

En referencia al trabajo de Machado et al. (2010) en el que se demostró la relación de dependencia entre la ansiedad y la baja calidad de vida percibida, el presente estudio encontró que la disponibilidad de recursos percibidos correlaciona indirectamente a la satisfacción de vida a través de las normas de contexto.

La calidad de vida al haber sido considerada desde dos dimensiones preponderantes: subjetiva y física, supuso la interrelación entre disponibilidad de recursos y procesos psicológicos básicos en los que los vínculos de grupos no incidirían sobre la percepción de satisfacción plena.

No obstante que en este trabajo las relaciones de confianza y la percepción de justicia son variables relativas a la dinámica de grupos a los que el individuo pertenece, su influencia es menor a la valoración de los recursos (servicios públicos de educación, tecnología y empleo) y las capacidades personales (libertades de elección, habilidades y conocimientos), aunque las normas de contexto (conductas arraigadas) incrementan la relación entre los recursos disponibles y la satisfacción de vida. Empero, será fundamental incluir las patologías personales en el modelo de relaciones de dependencia para contrastar su influencia en la satisfacción de vida.

\section{Conclusión}

La calidad de vida, en su dimensión de satisfacción plena de los servicios públicos de educación, Internet y empleo, en la muestra de jóvenes encuestados es: la relación indirecta entre los recursos percibidos y la satisfacción de vida sugiere que existen factores grupales y personales que regulan el impacto de una escasez o abundancia percibida de los recursos, aunque el estado del conocimiento advierte que son las variables psicológicas las que se asocian a las percepciones de satisfacción plena de la vida.

La satisfacción de vida al interrelacionarse con recursos percibidos, valoraciones del entorno, normas de contexto, percepción de justicia, relaciones de confianza, oportunidades y capacidades esperadas puede explicarse desde marcos teóricos psicológicos o sociológicos, pero es menester incluir teorías y constructos de orden económico y político, ya que su exclusión reduce la calidad de vida a la percepción de los encuestados.

\section{Referencias}

Abolfotouh, M., Salam, M., Alturaif, D., Suliman, W., Al-Essa, N., Al-issa, H., \& Al-rowaily, M. (2013). Predictors of quality of life and glycemic control among Saudi adults with diabetes. International Journal of Medicne and Medical Sciences, 46, 1360-1370. 
196 Cruz García Lirios - Javier Carreón Guillén - Jorge Hernández Valdés - Bertha Leticia Rivera Varela María Beatriz Castillo Escamilla - Erle García Estrada - José Francisco Rosas Ferrusca

Arístegui, I., \& Vázquez. (2013). El impacto del estigma y la discriminación en la calidad de vida de personas transgénero viviendo con VIH. Hologramática, 19, 5-30.

Baldi, G., \& García, E. (2010). Percepción de la calidad de vida en una muestra de invidividuos de la ciudad de San Luis, Argentina. Universidades, 40, 17-26.

Barranco, C., Delgado, M., Melin, C., \& Quintana, R. (2010). Trabajo social en vivienda: investigación sobre la calidad de vida percibida. Biblio, 2, 102-113.

Benites, L. (2010). Autismo, familia y calidad de vida. Cultura, 24, 1-20.

Carreón, J., \& García, C. (2013). Teorías de la seguridad pública y percepción del delito. Margen, 71, 1-16.

Carreón, J., García, C., Morales, M., Hernández, J., Rosas, J., \& Rivera, B. (2013). El desarrollo local sustentable en la esfera ciudadana. Economía y Sociedad, 18 (44), 35-48.

Derya, K. (2012). Genders differences on perceptions of employee quality for working life indicators in five star hotels in Turkey. International Journal of Academic research in Accounting, Finance and Management Sciences, 2, 195-203.

Ferragutti, G. (2012). Gubernamentalidad y capital humano. Hacia un esbozo de las condiciones de emergencia de los discursos sobre la sociedad de la información, educación y nuevas tecnologías. De Prácticas y Discursos, 1(1), 1-16.

García, C. (2013). Desarrollo local, vulnerabilidad hídrica, precariedad laboral, intensidad migratoria e identidad resiliente. Kairos, 32, 1-17.
García, C., Carreón, J., \& Hernández, J. (2014). La formación profesional del capital humano en la civilización del cambio climático. Revista Internacional de Investigación en Ciencias Sociales, 10(1), 107-125.

Grimaldo, M. (2010). Adaptación de la Escala de Calidad de Vida de Orson \& Barnes para profesionales de la salud. Cultura, 24, 1-20.

Machado, A., Anarte, M., \& Ruíz, M. (2010). Predictores de la calidad de vida en pacientes con diabetes mellitus tipo 1. Ciencia y Salud, 21, 35-47.

Melendro, E. (2011). La perspectiva ecosocial en la intervención socioeducativa con jóvenes excluidos. Un estudio comparado en $\mathrm{Ca}-$ nadá, Bélgica y España. Revista Española de Educación Comparada, 17, 197-218.

Picazo, E., Gutiérrez, E., Infante, J., \& Cantú, P. (2011). La Teoría del Desarrollo Humano y Sustentable: Hacia el reforzamiento de la salud como un derecho y libertad universal. Estudios Sociales, 19, 254-279.

Quiceno, J., \& Vinaccia, S. (2013). Resilencia, percepción de enfermedad, creencia, afrontamiento espiritual religioso y calidad de vida relacionada con la salud en pacientes con diagnóstico de artritis reumatoide. Psicología desde el Caribe, 30, 590-619.

Reyes, L. (2010). El dilema de los recursos naturales comunes. Gestión y Ambiente, 13, 71-80.

Sadeghzadeh, V. (2012). Improved quality of life with cardiac rehabilitation in post myocardial infarction patients. International Research Journal of Applied and Basic Sciences, 3, 394-401.

Tariq, Q. (2012). Impact of financial stress on life satisfaction. Asian Journal of Social Science \& Humanities, 1, 139-148. 